\title{
DNA FINGERPRINTING AND GENETIC DIVERSITIES IN SOME BANGLADESHI AUS RICE (Oryza sativa L.) GENOTYPES
}

\author{
M.M. Islam", H.A. Begum, M.S. Ali, M. Kamruzzaman \\ S. Hoque and M.I. Hoque \\ Bangladesh Institute of Nuclear Agriculture (BINA), Bangladesh Agricultural University \\ (BAU) campus, Mymensingh-2202, Bangladesh
}

\begin{abstract}
The allelic diversity and relationship among 120 Aus rice landraces were determined through DNA fingerprinting using microsatellite (SSR) markers. A total of 85 SSR markers were used to characterize and discriminate all tested Aus rice genotypes, 45 of which were polymorphic for different chromosome numbers. The number of alleles per locus varied from 6 alleles (RM484 and RM541) to 30 alleles (RM519) with an average of 13 alleles per locus. The polymorphic information content (PIC) values varied ranged from 0.5211 (RM536) to 0.9369 (RM519) with an average 0.8217 . The highest PIC value $(0.9369)$ was obtained for RM519 followed by RM286 (0.9357). The genetic distance-based results seen in the unrooted neighbor-joining tree clustering revealed nine genetic groups. Being grouped into distant clusters and with highest genetic distance, eleven genotypes viz., Atithi dhan, Kadar chap, Pankiraj, Japanese-7, Jamri saity, Logi jota, Joba, Lada moni, Manik Mondal-2, Boilum and Brmulka-2 could be selected as potential parents for crop improvement for their distinctive characters. Panchash and Parija had closest distance in the SSR based CS-Chord distance (0.000) might have same genetic background. The highest genetic dissimilarity (1.000) was found among the nineteen Aus genotypes combinations followed by the second highest (0.9778) among 94 Aus rice combinations. Whereas lowest genetic dissimilarity was found between Kala and Kalo Hizli (0.1778) followed by Holat and Holae $(0.2667)$. This information will be useful in the selection of diverse parents, background selection during backcross breeding programs and assist in broadening germplasm-based rice breeding programs in the near future.
\end{abstract}

Keywords: Aus rice, genetic diversity, microsatellite markers, DNA fingerprinting

\footnotetext{
"Corresponding author email: mislambrri73@gmail.com
} 


\section{INTRODUCTION}

Rice (Oryza sativa) is one of the most important food crops and a primary source of food for more than half of the world's population (Khush, 2005). According to the United Nations (UN) estimates, the current world population 6.1 billion is expected to reach 8.0 billion by 2025. Most of this increase (93\%) will take place in the developing world. Global rice production must reach 800 million tones of paddy rice to meet projected demand in 2025 (Peng et al., 1999) which is about 200 million tones more than rice production in 2006. This additional rice must come mainly from irrigated land in Asia, because improving rice yield in most rainfed regions is constrained by drought, flooding and poor soil quality (Cassman, 1999). Bangladesh is already under pressure both from huge and increasing demands for food, and from problems of agricultural land and water resources depletion. Bangladesh needs to increase the rice yield in order to meet the growing demand for food emanating from population growth.

For the study of genetic diversity, the plant scientists have used generally morphological, physiological as well as chemical features of plant. The number of scoreable morphological characters is varying as compared to the biological active genes. Moreover in most cases, plant genomes have large amount of repetitive DNA which are not expressed and do not contribute to the physiological or morphological appearance of plants. In the case of very closely related plant varieties, there are very few morphological differences, which as a matter of fact do not represent the true genetic differences at DNA level. So, there is always a need to study polymorphism at DNA level, which can be an indicative of genetic diversity. Several types of molecular markers viz., RFLP, RAPD, AFLP, microsatellites and SNP have been developed. PCR-based markers such as microsatellites are co-dominant, hyper variable, abundant and well distributed throughout the rice genome (Temnykh et al., 2001). Microsatellites have shown great promise in genetic diversity, genome mapping, gene tagging and marker-assisted selection (MAS) because they are technically simple, time saving, highly informative and require small amount of DNA. Abundance of microsatellite markers is now available through the published high-density linkage map (McCouch et al., 2002; IRGSP 2005) or public database. A study was conducted on 234 rice landraces in Plant breeding division, Cornell University and they identified five distinct groups corresponding to indica, aus, aromatic, temperate japonica and tropical japonica rice (Amanda et al., 2004). They also have very high diversity with $98 \%$ of loci polymorphic in Aus groups. Despite of their drought tolerance and early maturity, the group has received less attention compared to indica and japonica group.

There are four distinct ecotypes of rice-Boro, Aus, Transplanted aman and Deep water aman in Bangladesh. Bangladesh has a good source of indigenous rice

cultivars. About 4000 T. Aman, 2500 Boro and 1500 Aus landraces are present in BRRI rice germplasm gene bank. Only a few decades ago large numbers of farmers 
were growing local cultivars as their main crop. Those cultivars have good adaptation but are poor yielder. Actually cultivation of these landraces was gradually replaced by high yielding varieties during last twenty years. These landraces adapted in different parts of this country, some of which have very nice quality, fineness, aroma, taste and high protein contents (Dutta et al., 1998). After establishment of BRRI, DNA fingerprinting has been done only for a small number of local germplasm. Indigenous crop landraces were characterized at both molecular and phenotypic level by many countries. Such types of characterization have been done for keeping the crop identity and searching for new genes for further crop improvement. But information on the genetic diversity of local landraces particularly for Aus rice is very scanty. Precise information on the extent of genetic diversity among population is crucial in any crop improvement program, as selection of plants based on genetic diversity has become successful in several crops (Ananda and Rawat, 1984; De et al., 1988). That's why, the present investigation has been undertaken in order to find out the genetic diversities among Aus genotypes at the molecular level.

\section{MATERIALS AND METHODS}

\section{Plant materials and genomic DNA isolation}

One hundred and twenty genotypes, including twelve BRRI released Aus genotypes were used in this study (Table 1). Genomic DNA was isolated from young leaves from 21 days old plants with minor modification of CTAB method. The concentration of extracted DNA was estimated by DNA confirmation test by (1.5\%) agarose gel electrophoresis with lambda DNA (50ng/ $\mu$ l).

Table 1. List of one hundred and twenty Aus rice genotypes

\begin{tabular}{l|l|c|l|l|l|l|l|l|l}
\hline SL\# & $\begin{array}{c}\text { Name of } \\
\text { Genotypes }\end{array}$ & $\begin{array}{c}\text { Acc } \\
\text { No. }\end{array}$ & Origin & Collection & SL\# & $\begin{array}{c}\text { Name of } \\
\text { Genotypes }\end{array}$ & $\begin{array}{c}\text { Acc } \\
\text { No. }\end{array}$ & Origin & Collection \\
\hline 1 & Ajab Bett & 1546 & Chittagong & BRRI & 61 & Kheri Jamri & 4029 & Kushtia & BRRI \\
2 & Agun Ban & 1770 & Jessore & BRRI & 62 & Khamar Mundu & 4040 & Meharpur & BRRI \\
3 & Atithi dhan & 4568 & Dhaka & BRRI & 63 & Kaika & 4041 & Meharpur & BRRI \\
4 & Aalo Sate & 4752 & Feni & BRRI & 64 & Kadar Chap & 4042 & Meharpur & BRRI \\
5 & Begun Bahar & 651 & Comilla & BRRI & 65 & Laksmi lofa & 1211 & Faridpur & BRRI \\
6 & Boilum & 1205 & Faridpur & BRRI & 66 & Lada Moni & 1286 & Kushtia & BRRI \\
7 & Barmulka-2 & 1212 & Faridpur & BRRI & 67 & Lagi jota & 1768 & Jessore & BRRI \\
8 & Benaful & 1529 & Dinajpur & BRRI & 68 & Manik Modu & 1323 & Kushtia & BRRI \\
9 & Benaful & 1773 & Jessore & BRRI & 69 & Mary satia & 1626 & Comilla & BRRI \\
10 & Bathuri & 1550 & Chittagong & BRRI & 70 & Manik Mondal-1 & 1692 & Faridpur & BRRI \\
11 & Baisamugur & 1696 & Faridpur & BRRI & 71 & Manik Mondol-2 & 1765 & Jessore & BRRI \\
12 & Baismuguria & 1701 & Faridpur & BRRI & 72 & Madhu Mala & 1737 & Khulna & BRRI \\
13 & Bawoi & 1721 & Khulna & BRRI & 73 & Maraka & 2316 & Dhaka & BRRI \\
& & & & & & Migichak & & & \\
\hline
\end{tabular}




\begin{tabular}{|c|c|c|c|c|c|c|c|c|c|}
\hline SL\# & $\begin{array}{c}\text { Name of } \\
\text { Genotypes }\end{array}$ & $\begin{array}{l}\text { Acc } \\
\text { No. }\end{array}$ & Origin & Collection & SL\# & $\begin{array}{c}\text { Name of } \\
\text { Genotypes }\end{array}$ & $\begin{array}{l}\text { Acc } \\
\text { No. }\end{array}$ & Origin & Collection \\
\hline 14 & Beri & 1751 & Khulna & BRRI & 74 & Mazra & 4019 & Faridpur & BRRI \\
\hline 15 & Beni muri & 1767 & Jessore & BRRI & 75 & Magi Sarsa & 4033 & Jessore & BRRI \\
\hline 16 & BR319-1-HR-12 & 3843 & Dhaka & BRRI & 76 & Moush Doll & 4036 & Jessore & BRRI \\
\hline 17 & Bora dhan & 4020 & Kustia & BRRI & 77 & Morich Boti & 4043 & Meharpur & BRRI \\
\hline 18 & Baisha Muri & 4026 & Faridpur & BRRI & 78 & Mi-Mandi & 4573 & Dhaka & BRRI \\
\hline 19 & $\mathrm{Bar} \mathrm{Pa}$ & 4027 & Jhenaidhah & BRRI & 79 & Mi-mandisarang & 4574 & Dhaka & BRRI \\
\hline 20 & Balion & 4032 & Faridpur & BRRI & 80 & Matia & 4596 & Noakhali & BRRI \\
\hline 21 & Bil Kalae & 4038 & Jessore & BRRI & 81 & Nayan Tara & 654 & Comilla & BRRI \\
\hline 22 & Balam & 4045 & Kustia & BRRI & 82 & Nusha Ratoi & 4046 & Khulna & BRRI \\
\hline 23 & Bhatkarari & 4551 & Dhaka & BRRI & 83 & Nordi & 4751 & Jessore & BRRI \\
\hline 24 & Boailla & 4559 & Dhaka & BRRI & 84 & Porangi 7 & 1216 & Faridpur & BRRI \\
\hline 25 & Borga Dhan & 4565 & Dhaka & BRRI & 85 & Parangi & 1689 & Faridpur & BRRI \\
\hline 26 & Bali Bokri & 4587 & Dhaka & BRRI & 86 & Paik jota & 1528 & Dinajpur & BRRI \\
\hline 27 & Chenri & 808 & Sylhet & BRRI & 87 & Pankirajs & 1700 & Faridpur & BRRI \\
\hline 28 & Chamka & 1549 & Chittagong & BRRI & 88 & Pipre Sail & 1723 & Khulna & BRRI \\
\hline 29 & Chiknal & 1642 & Noakhali & BRRI & 89 & Panburi & 1730 & Khulna & BRRI \\
\hline 30 & Chitri & 2081 & Dhaka & BRRI & 90 & Padma Moni & 1782 & Jessore & BRRI \\
\hline 31 & Chapila & 4571 & Dhaka & BRRI & 91 & Padha Moidu & 4039 & Meharpur & BRRI \\
\hline 32 & Chakulya & 4575 & Dhaka & BRRI & 92 & Panchash & 4566 & Dhaka & BRRI \\
\hline 33 & Darial & 649 & Comilla & BRRI & 93 & Parija & 4588 & Feni & BRRI \\
\hline 34 & Goreswar & 953 & Faridpur & BRRI & 94 & Ratol & 1772 & Jessore & BRRI \\
\hline 35 & Gutle & 1774 & Jessore & BRRI & 95 & Rathail & 4047 & Bagarhat & BRRI \\
\hline 36 & Hidi 2 & 1289 & Kushtia & BRRI & 96 & Saita & 1681 & Faridpur & BRRI \\
\hline 37 & Holat & 1551 & Chittagong & BRRI & 97 & Sribalium & 1699 & Faridpur & BRRI \\
\hline 38 & Holae & 1656 & Dhaka & BRRI & 98 & Soloi & 1720 & Khulna & BRRI \\
\hline 39 & Haita saita & 1691 & Faridpur & BRRI & 99 & Sodai Soru & 1725 & Khulna & BRRI \\
\hline 40 & Honuman jota & 1739 & Khulna & BRRI & 100 & Saribail & 1756 & Jessore & BRRI \\
\hline 41 & Hijoli Aus & 4048 & Pabna & BRRI & 101 & Soda & 1762 & Jessore & BRRI \\
\hline 42 & Haji Sail & 4564 & Dhaka & BRRI & 102 & Sail bogi & 2077 & Dhaka & BRRI \\
\hline 43 & Hati Bajor & 4766 & Bagerhat & BRRI & 103 & Tarabali & 811 & Sylhet & BRRI \\
\hline 44 & IR19746-28-2-2 & 3821 & Dhaka & BRRI & 104 & Tepakain & 1532 & Dinajpur & BRRI \\
\hline 45 & Jamri saity & 1317 & Kushtia & BRRI & 105 & Tapa sail & 1752 & Khulna & BRRI \\
\hline 46 & Jamurus & 1525 & Dinajpur & BRRI & 106 & Tusha & 4567 & Dhaka & BRRI \\
\hline 47 & Jagli & 1761 & Jessore & BRRI & 107 & Udobali & 4572 & Dhaka & BRRI \\
\hline 48 & Japanese \#7 & 3611 & Japanese & BRRI & 108 & Zamir Saita & 4044 & Meharpur & BRRI \\
\hline 49 & Japanese \#3 & 3617 & Japanese & BRRI & 109 & BR1(chandina) & $M V^{*}$ & - & BRRI \\
\hline 50 & Joba & 4030 & Kushtia & BRRI & 110 & BR2 (Mala) & MV & - & BRRI \\
\hline 51 & Korcha Muri & 948 & Khulna & BRRI & 111 & BR3(Biplob) & MV & - & BRRI \\
\hline 52 & Khushni & 952 & Khulna & BRRI & 112 & BR6 & MV & - & BRRI \\
\hline
\end{tabular}




\begin{tabular}{|c|c|c|c|c|c|c|c|c|c|}
\hline SL\# & $\begin{array}{c}\text { Name of } \\
\text { Genotypes }\end{array}$ & $\begin{array}{l}\text { Acc } \\
\text { No. }\end{array}$ & Origin & Collection & SL\# & $\begin{array}{c}\text { Name of } \\
\text { Genotypes }\end{array}$ & $\begin{array}{l}\text { Acc } \\
\text { No. }\end{array}$ & Origin & Collection \\
\hline 53 & Katar & 1632 & Chittagong & BRRI & 113 & $\begin{array}{l}\text { BR7 (Brri } \\
\text { Balam) }\end{array}$ & MV & - & BRRI \\
\hline 54 & Kali Bori & 1633 & Jessore & BRRI & 114 & BR8 (Aasa) & MV & - & BRRI \\
\hline 55 & Kamani sail & 1662 & $\begin{array}{l}\text { Chitta. H. } \\
\mathrm{T}\end{array}$ & BRRI & 115 & BR9 (Sufala) & MV & - & BRRI \\
\hline 56 & Kali Boro & 1707 & Faridpur & BRRI & 116 & BR12 (Mayana) & MV & - & BRRI \\
\hline 57 & Koblerash & 1728 & Khulna & BRRI & 117 & BR15 (Mohinye) & MV & - & BRRI \\
\hline 58 & Korcha & 1755 & Jessore & BRRI & 118 & $\begin{array}{l}\text { BR16 (Shahi } \\
\text { Balam) }\end{array}$ & MV & - & BRRI \\
\hline 59 & Kala & 4023 & Jessore & BRRI & 119 & BR20 (Nizamy) & MV & - & BRRI \\
\hline 60 & Kalo Hizli & 4025 & Kushtia & BRRI & 120 & BR21 (Niamat) & MV & - & BRRI \\
\hline
\end{tabular}

* = Modern BRRI released Aus Variety; \# = S1 No. 7-94 local Aus landraces

\section{SSR primers analysis}

A total of 45 primers were selected (Table 2) to detect polymorphic DNA alleles for discriminating the tested Aus rice genotypes. Each PCR was carried out in a $10 \mu \mathrm{l}$ reaction volume containing $1 \mu \mathrm{l}$ of $\mathrm{MgCl}_{2}$ free $10 \mathrm{X}$ PCR buffer with $\left(\mathrm{NH}_{4}\right)_{2} \mathrm{SO}_{4}, 1.2 \mu \mathrm{l}$ of $25 \mathrm{mM} \mathrm{MgCl} 2,0.2 \mu \mathrm{l}$ of $10 \mathrm{mM}$ dNTPs, $0.2 \mu \mathrm{l}$ of $5 \mathrm{U} / \mu \mathrm{l} \mathrm{Taq}$ DNA polymerase, $0.5 \mu \mathrm{l}$ of $10 \mu \mathrm{M}$ forward and reverse primers and $3 \mu \mathrm{l}$ (10ng) of DNA using a 96 well thermal cycler. The mixture was overlaid with one drop $(3 \mu \mathrm{l})$ of mineral oil to prevent evaporation. The temperature profile used for PCR amplification comprised $94^{\circ} \mathrm{C}$ for 5 minutes (initial denaturation) followed by 35 cycles of $94^{\circ} \mathrm{C}$ for 1 minute (denaturation), $55^{\circ} \mathrm{C}$ for 1 minute (annealing), $72^{\circ} \mathrm{C}$ for 2 minutes (extension) with a final extension for 7 minutes at $72^{\circ} \mathrm{C}$ at the end of 35 cycles. The annealing temperatures were adjusted based on the specific requirements of each primer combination. The PCR products were mixed with gel loading dye (bromophenol blue, xylene cyanol and sucrose) and electrophoresed in $8 \%$ polyacrylamide gel using vertical poly acrylamide gels for high throughput manual genotyping. Three-Four $\mu 1$ of amplification products were resolved by running gel in $1 \mathrm{X}$ TBE buffer for $1.5 \mathrm{hrs}$ to $2.5 \mathrm{hrs}$ depending upon the allele size at around 90 volts and $500 \mathrm{~mA}$ electricity. The gels were stained in $1 \mu \mathrm{g} / \mathrm{ml}$ ethidium bromide and were documented using UVPRO (Uvipro Platinum, EU) gel documentation unit.

\section{Data analysis}

Size for each amplified allele was measured in base pair using Alpha-EaseFC 5.0 software. The summary statistics including the number of alleles per locus, major allele frequency, gene diversity, Polymorphism Information Content (PIC) values were determined using Power Marker version 3.25 (Liu and Muse, 2005). The allele frequency data from Power Marker was used to export in binary format (allele 
presence $=1$ and allele absence $=0$ ) for analysis with NTSYS-pc version 2.1 (Rohlf, 2002). A similarity matrix was calculated with the Simqual subprogram using the Dice coefficient, followed by cluster analysis with the SAHN subprogram using the UPGMA clustering method as implemented in NTSYS-pc.

Table 2. Selected primers, their sequence and chromosome number

\begin{tabular}{|c|c|c|c|c|c|}
\hline $\begin{array}{l}\text { Primer } \\
\text { code }\end{array}$ & Chr No & $\begin{array}{c}\text { Position } \\
\text { (cM) }\end{array}$ & $\begin{array}{l}\text { Product } \\
\text { Size(bp) }\end{array}$ & Forward primer sequence ( $5^{\prime}$ to $3^{\prime}$ ) & Reverse primer sequence ( $5^{\prime}$ to $3^{\prime}$ ) \\
\hline RM1 & 1 & 29.7 & 113 & GCGAAAACACAATGCAAAAA & GCGTTGGTTGGACCTGAC \\
\hline RM283 & 1 & 31.4 & 151 & GTCTACATGTACCCTTGTTGGG & CGGCATGAGAGTCTGTGATG \\
\hline RM237 & 1 & 115.2 & 130 & CAAATCCCGACTGCTGTCC & TGGGAAGAGAGCACTACAGC \\
\hline RM259 & 1 & 54.2 & 162 & TGGAGTTTGAGAGGAGGG & CTTGTTGCATGGTGCCATGT \\
\hline RM431 & 1 & 178.3 & 251 & TCCTGCGAACTGAAGAGTTG & AGAGCAAAACCCTGGTTCAC \\
\hline RM452 & 2 & 58.4 & 209 & CTGATCGAGAGCGTTAAGGG & GGGATCAAACCACGTTTCTG \\
\hline RM154 & 2 & 4.8 & 183 & АСССТCTCCGCCTCGCCTCCTC & СТCCTCCTCCTGCGACCGCTCC \\
\hline RM327 & 2 & 67.45 & 213 & СТАСТССТСТGTCССТССТСТС & CCAGCTAGACACAATCGAGC \\
\hline RM514 & 3 & 216.4 & 259 & AGATTGATCTCCCATTCCCC & CACGAGCATATTACTAGTGG \\
\hline RM489 & 3 & 29.2 & 271 & ACTTGAGACGATCGGACACC & TCACCCATGGATGTTGTCAG \\
\hline RM85 & 3 & 231 & 107 & CCAAAGATGAAACCTGGATTG & GCACAAGGTGAGCAGTCC \\
\hline RM307 & 4 & 0 & 174 & GTACTACCGACCTACCGTTCAC & CTGCTATGCATGAACTGCTC \\
\hline RM252 & 4 & 99 & 216 & TTCGCTGACGTGATAGGTTG & ATGACTTGATCCCGAGAACG \\
\hline RM119 & 4 & 76.1 & 166 & CATCCCCCTGCTGCTGCTGCTG & CGCCGGATGTGTGGGACTAGCG \\
\hline RM178 & 5 & 118.8 & 117 & CAGTGGGCGAGCATAGGAG & АТССТTTтСтCССТCTCTCG \\
\hline RM413 & 5 & 26.7 & 79 & GGCGATTCTTGGATGAAGAG & TCCCCACCAATCTTGTCTTC \\
\hline RM169 & 5 & 57.9 & 167 & TGGCTGGCTCCGTGGGTAGCTG & TCCCGTTGCCGTTCATCCCTCC \\
\hline RM153 & 5 & $0-2.3$ & 201 & ACCAACGCCAAAAGCTACTG & TACTCGCCCTGCATGAGC \\
\hline RM122 & 5 & 6.4 & 227 & GAGTCGATGTAATGTCATCAGTGC & GAAGGAGGTATCGCTTTGTTGGAC \\
\hline RM161 & 5 & 96.9 & 187 & AAACTGTTTTACCCCTGGCC & ATCCCCTTCTGCGGTAAAAC \\
\hline RM541 & 6 & 75.5 & 158 & TATAACCGACCTCAGTGCCC & ССTTACTCCCATGCCATGAG \\
\hline RM204 & 6 & 25.1 & 169 & GTGACTGACTTGGTCATAGGG & GCTAGCCATGCTCTCGTACC \\
\hline RM217 & 6 & 28.6 & 133 & ATCGCAGCAATGCCTCGT & GGGTGTGAACAAAGACAC \\
\hline RM11 & 7 & 47 & 140 & TCTCCTCTTCCCCCGATC & ATAGCGGGCGAGGCTTAG \\
\hline RM18 & 7 & 90.4 & 157 & TTCCСТCTCATGAGCTCCAT & GAGTGCCTGGCGCTGTAC \\
\hline RM134 & 7 & 73.2 & 93 & ACAAGGCCGCGAGAGGATTCCG & GCTCTCCGGTGGCTCCGATTGG \\
\hline RM25 & 8 & 52.2 & 146 & GGAAAGAATGATCTTTTCATGG & CTACCATCAAAACCAATGTTC \\
\hline RM44 & 8 & 60.9 & 99 & ACGGGCAATCCGAACAACC & TCGGGAAAACCTACCCTACC \\
\hline RM105 & 9 & 32.1 & 134 & GTCGTCGACCCATCGGAGCCAC & TGGTCGAGGTGGGGATCGGGTC \\
\hline RM215 & 9 & 99.4 & 148 & CAAAATGGAGCAGCAAGAGC & TGAGCACCTCCTTCTCTGTAG \\
\hline RM219 & 9 & 11.7 & 202 & CGTCGGATGATGTAAAGCCT & CATATCGGCATTCGCCTG \\
\hline RM171 & 10 & 58.1 & 328 & AACGCGAGGACACGTACTTAC & ACGAGATACGTACGCCTTTG \\
\hline RM147 & 10 & 99.8 & 97 & TACGGCTTCGGCGGCTGATTCC & CCCCCGAATCCCATCGAAACCC \\
\hline RM484 & 10 & 97.3 & 299 & ТСТСССТССТСАССАТТGTC & TGCTGCCCTCTCTCTCTCTC \\
\hline RM216 & 10 & 17.6 & 146 & GCATGGCCGATGGTAAAG & TGTATAAAACCACACGGCCA \\
\hline RM536 & 11 & 55.1 & 243 & TCTCTCCTCTTGTTTGGCTC & ACACACCAACACGACCACAC \\
\hline
\end{tabular}




\begin{tabular}{c|c|c|c|l|l}
\hline $\begin{array}{c}\text { Primer } \\
\text { code }\end{array}$ & Chr No & $\begin{array}{c}\text { Position } \\
\text { (cM) }\end{array}$ & $\begin{array}{c}\text { Product } \\
\text { Size(bp) }\end{array}$ & Forward primer sequence (5' to 3') & \multicolumn{1}{|c}{ Reverse primer sequence (5' to 3') } \\
\hline RM209 & 11 & 73.9 & 134 & ATATGAGTTGCTGTCGTGCG & CAACTTGCATCCTCCCCTCC \\
RM167 & 11 & 73.9 & 128 & GATCCAGCGTGAGGAACACGT & AGTCCGACCACAAGGTGCGTTGTC \\
RM206 & 11 & 102.9 & 147 & CCCATGCGTTTAACTATTCT & CGTTCCATCGATCCGTATGG \\
RM286 & 11 & 0 & 110 & GGCTTCATCTTTGGCGAC & CCGGATTCACGAGATAAACTC \\
RM144 & 11 & 123.2 & 237 & TGCCCTGGCGCAAATTTGATCC & GCTAGAGGAGATCAGATGGTAGTGC \\
RM287 & 11 & 68.6 & 118 & TTCCCTGTTAAGAGAGAAATC & GTGTATTTGGTGAAAGCAAC \\
RM20 & 12 & 0 & 234 & ATCTTGTCCCTGCAGGTCAT & GAAACAGAGGCACATTTCATTG \\
RM519 & 12 & 62.6 & 122 & AGAGAGCCCCTAAATTTCCG & AGGTACGCTCACCTGTGGAC \\
RM277 & 12 & 57.2 & 124 & CGGTCAAATCATCACCTGAC & CAAGGCTTGCAAGGGAAG \\
\hline
\end{tabular}

\section{RESULTS}

\section{Overall microsatellite diversity}

One hundred and twenty Aus genotypes were assessed for genetic variability using 45 polymorphic Microsatellite DNA markers. A total of 228 alleles were detected at the loci of 45 microsatellite markers across the Aus rice genotypes.

Table 3. Data on the number of alleles, allele size range, highest frequency allele and polymorphism information content (PIC)

\begin{tabular}{|c|c|c|c|c|c|c|c|c|}
\hline \multirow[t]{2}{*}{ Marker } & \multirow[t]{2}{*}{$\begin{array}{l}\mathrm{Chr} \\
\text { No }\end{array}$} & \multirow[t]{2}{*}{$\begin{array}{l}\text { Position } \\
\text { (cM) }\end{array}$} & \multirow[t]{2}{*}{ Motif* } & \multirow[t]{2}{*}{ Allele no. } & \multirow[t]{2}{*}{$\begin{array}{l}\text { Size range } \\
\text { (bp) }\end{array}$} & \multicolumn{2}{|c|}{$\begin{array}{l}\text { Highest frequency } \\
\text { allele }\end{array}$} & \multirow[t]{2}{*}{ PIC Value } \\
\hline & & & & & & Size (bp) & Freq $(\%)$ & \\
\hline RM1 & 1 & 29.7 & (GA)26 & 19 & $70-121$ & 79 & 16 & 0.9052 \\
\hline RM283 & 1 & 31.4 & $(\mathrm{GA}) 18$ & 13 & $146-170$ & 155 & 33 & 0.8172 \\
\hline RM237 & 1 & 115.2 & (CT) 18 & 7 & $136-150$ & 140 & 36 & 0.7146 \\
\hline RM259 & 1 & 54.2 & (CT) 17 & 16 & $152-175$ & 168 & 23 & 0.8672 \\
\hline RM431 & 1 & 178.3 & (AG) 16 & 17 & $235-270$ & 262 & 13 & 0.9154 \\
\hline RM452 & 2 & 58.4 & (GTC)9 & 12 & $185-217$ & 190,197 & 17 & 0.8760 \\
\hline RM154 & 2 & 4.8 & $(\mathrm{GA}) 21$ & 25 & $160-220$ & 190 & 15 & 0.9161 \\
\hline RM327 & 2 & 67.45 & (CAT)11(CTT)5 & 14 & $193-216$ & 215 & 16 & 0.8890 \\
\hline RM514 & 3 & 216.4 & (AC) 12 & 9 & $245-262$ & 245,252 & 18 & 0.8475 \\
\hline RM489 & 3 & 29.2 & $(\mathrm{ATA}) 8$ & 18 & $236-271$ & 254 & 18 & 0.8753 \\
\hline RM85 & 3 & 231 & (TGG)5(TCT) 12 & 7 & $89-117$ & 107 & 35 & 0.7062 \\
\hline RM307 & 4 & 0 & (AT)14(GT)21 & 18 & $129-186$ & 148 & 23 & 0.8880 \\
\hline RM252 & 4 & 99 & (CT) 19 & 16 & $198-245$ & 203 & 21 & 0.8829 \\
\hline RM119 & 4 & 76.1 & (GTC)6 & 10 & $160-174$ & 160 & 18 & 0.8508 \\
\hline RM178 & 5 & 118.8 & $(\mathrm{GA}) 5(\mathrm{AG}) 8$ & 9 & $117-124$ & 124 & 33 & 0.7682 \\
\hline RM413 & 5 & 26.7 & (AG)11 & 14 & $70-101$ & 82 & 19 & 0.8705 \\
\hline RM169 & 5 & 57.9 & $(\mathrm{GA}) 12$ & 19 & $163-219$ & 204 & 12 & 0.9211 \\
\hline RM153 & 5 & $0-2.3$ & $($ GAA $) 9$ & 25 & $177-230$ & 218 & 14 & 0.9332 \\
\hline
\end{tabular}




\begin{tabular}{|c|c|c|c|c|c|c|c|c|}
\hline \multirow[t]{2}{*}{ Marker } & \multirow[t]{2}{*}{$\begin{array}{l}\text { Chr } \\
\text { No }\end{array}$} & \multirow[t]{2}{*}{$\begin{array}{l}\text { Position } \\
\text { (cM) }\end{array}$} & \multirow[t]{2}{*}{ Motif* } & \multirow[t]{2}{*}{ Allele no. } & \multirow[t]{2}{*}{$\begin{array}{l}\text { Size range } \\
\text { (bp) }\end{array}$} & \multicolumn{2}{|c|}{$\begin{array}{l}\text { Highest frequency } \\
\text { allele }\end{array}$} & \multirow[t]{2}{*}{ PIC Value } \\
\hline & & & & & & Size (bp) & Freq $(\%)$ & \\
\hline RM122 & 5 & 6.4 & $\begin{array}{c}(\mathrm{GA}) 7 \mathrm{~A}(\mathrm{GA}) 2 \mathrm{~A}( \\
\mathrm{GA}) 11\end{array}$ & 8 & $223-238$ & 227 & 28 & 0.8032 \\
\hline RM161 & 5 & 96.9 & (AG)20 & 8 & $165-186$ & 172 & 46 & 0.6917 \\
\hline RM541 & 6 & 75.5 & (TC) 16 & 6 & $158-170$ & 168 & 28 & 0.7671 \\
\hline RM204 & 6 & 25.1 & (CT)44 & 16 & $106-155$ & 115 & 22 & 0.8639 \\
\hline RM217 & 6 & 28.6 & (CT) 20 & 11 & $124-155$ & 134,137 & 23 & 0.8080 \\
\hline RM11 & 7 & 47 & (GA)17 & 8 & $123-147$ & 124 & 48 & 0.6227 \\
\hline RM18 & 7 & 90.4 & $\begin{array}{c}(\mathrm{GA}) 4 \mathrm{AA}(\mathrm{GA})(\mathrm{A} \\
\mathrm{G}) 16\end{array}$ & 11 & $153-171$ & 160 & 32 & 0.7831 \\
\hline RM134 & 7 & 73.2 & $(\mathrm{CCA}) 7$ & 7 & $82-94$ & 93 & 21 & 0.8272 \\
\hline RM25 & 8 & 52.2 & (GA)18 & 14 & $124-158$ & 135 & 29 & 0.8477 \\
\hline RM44 & 8 & 60.9 & (GA) 16 & 14 & $104-120$ & 111 & 13 & 0.8907 \\
\hline RM105 & 9 & 32.1 & $(\mathrm{CCT}) 6$ & 13 & $130-144$ & 135 & 21 & 0.8539 \\
\hline RM215 & 9 & 99.4 & (CT)16 & 11 & $144-168$ & 147 & 25 & 0.8120 \\
\hline RM219 & 9 & 11.7 & (CT)17 & 14 & $195-230$ & 210 & 17 & 0.8834 \\
\hline RM171 & 10 & 58.1 & (GATG)5 & 18 & $289-334$ & 317 & 15 & 0.8907 \\
\hline RM147 & 10 & 99.8 & (TTCC)5(GGT)5 & 7 & $93-99$ & 95 & 37 & 0.7036 \\
\hline RM484 & 10 & 97.3 & $(\mathrm{AT}) 9$ & 6 & $290-319$ & 299 & 48 & 0.6233 \\
\hline RM216 & 10 & 17.6 & (CT) 18 & 12 & $122-147$ & 128 & 37 & 0.7112 \\
\hline RM536 & 11 & 55.1 & (CT)16 & 9 & $238-252$ & 250 & 62 & 0.5211 \\
\hline RM209 & 11 & 73.9 & (CT) 18 & 17 & $122-160$ & 154 & 18 & 0.8779 \\
\hline RM167 & 11 & 73.9 & (CT) 18 & 10 & $124-159$ & 147 & 28 & 0.8056 \\
\hline RM206 & 11 & 102.9 & (CT) 21 & 14 & $126-171$ & 132 & 24 & 0.8478 \\
\hline RM286 & 11 & 0 & $(\mathrm{GA}) 16$ & 25 & $96-130$ & 117 & 13 & 0.9357 \\
\hline RM144 & 11 & 123.2 & $(\mathrm{ATT}) 11$ & 24 & $214-261$ & 241 & 15 & 0.9218 \\
\hline RM287 & 11 & 68.6 & $(\mathrm{GA}) 21$ & 14 & $96-119$ & 103 & 28 & 0.8382 \\
\hline RM20 & 12 & 0 & $(\mathrm{ATT}) 14$ & 9 & $155-191$ & 165 & 25 & 0.8078 \\
\hline RM519 & 12 & 62.6 & $(\mathrm{AAG}) 8$ & 30 & $117-150$ & 129 & 10 & 0.9369 \\
\hline \multirow[t]{2}{*}{ RM277 } & 12 & 57.2 & (GA)11 & 7 & $116-124$ & 124 & 37 & 0.6556 \\
\hline & & & & 13 & & & 25 & 0.8217 \\
\hline
\end{tabular}

* Motif of the SSR and number of repeats as previously published (http://www.gramene.org)

The highest amplicon size was produced by RM171 (334 bp) and the lowest by RM1 (70 bp). The number of alleles per locus ranged from 6 alleles (RM484 and RM541) to 30 alleles (RM519), with an average of 13 alleles across the 45 loci (Table 3). The frequency of the most common allele at each locus ranged from $10 \%$ (RM519) to 62\% (RM536). On average, 25\% of the 120 Aus rice genotypes shared a common major allele at any given locus. The polymorphic information content (PIC) 
values were ranged 0.5211 (RM536) to 0.9369 (RM519) with an average 0.8217 . The highest PIC value (0.9369) was obtained for RM519 followed by RM286 (0.9357), RM153 (0.9332), RM144 (0.9218) and RM169 (0.9211), respectively. PIC value revealed that RM519 and RM286 were considered as the best marker for 120 Aus genotypes. The gel pictures of figure 1 showed amplified fragment using primer designed for the SSR marker RM519 for all 120 genotypes.

Table 4. 100\% dissimilarity of the nineteen Aus genotypes

\begin{tabular}{ll}
\hline S1 \# & Combinations \\
\hline 1 & IR19746-28-2-2 $\times$ Tapa sail \\
2 & Holae $\times$ Sail bogi \\
3 & IR19746-28-2-2XUdobali \\
4 & IR19746-28-2-2 $\times$ Zamir Saita \\
5 & Jamri saity $\times$ BR6 \\
6 & Boilum $\times$ BR6 \\
7 & Barmulka- $\times$ Soda \\
8 & Mi-Mandi $\times$ Baisamugur \\
9 & Baismuguria X Lagi jota \\
10 & Beni muri $\times$ Lakhi Lata \\
11 & Bar Pa $\times$ Noroi \\
12 & Balam $\times$ Noroi \\
13 & Bil Kalae $\times$ Padha Moidu \\
14 & Bil Kalae $\times$ Panchash \\
15 & Balam $\times$ Padha Moidu \\
16 & Balam $\times$ Panchash \\
17 & Darial $\times$ Parija \\
18 & Holae $\times$ Parija \\
19 & Hati Bajor $\times$ Sodai Soru \\
\hline
\end{tabular}

\section{Genetic distance-based analysis}

An unrooted neighbor-joining tree showing the genetic relationships among 120 Aus rice genotypes of Bangladesh was constructed based on the alleles detected by 45 microsatellite markers. The genetic distance-based results seen in the unrooted neighbor-joining tree revealed nine groups in the 120 genotypes (Figure 2). Aus genotypes of BRRI released modern varieties were clustered in the same group in the cluster IX. All Aus landraces were distributed into different cluster but Panchash (sl no.92, acc. no. 4039) were not found in any cluster, it may be duplicate with Parija (sl 
no. 93, acc. no.4566). Cluster number VIII contain highest number of genotypes (23) and cluster no IV contain only one genotypes, it was Jamri saity. Furthermore, the two Aus landraces viz., Madhu Mala (73) and Khushni (52) were clustered in the same group (cluster II). Three Aus landraces (Hati Bajor, Haji Sail, and IR19746-282-2) were clustered distinctly in the same group (cluster VII). Cluster III and Cluster $\mathrm{V}$ contains same number of genotypes (21) on the other hand cluster I and cluster VI contain 17 and 19 number of genotypes, respectively. Genetic dissimilarity coefficient was recognized between every two genotypes based on DNA profile. The highest genetic dissimilarity (1.000) was found among the nineteen Aus genotypes combinations (Table 4.) Followed by the second highest (0.9778) ninety four Aus rice combinations (Table 5). Whereas lowest (0.1778) genetic dissimilarity was found Kala and Kalo Hizli.

Table 5. 98\% dissimilarity found in 98 combinations of Aus genotypes

\begin{tabular}{llll}
\hline S1 \# & Combinations & S1 \# & Combinations \\
1 & Bathuri $\times$ BR6 & 48 & Bil Kalae $\times$ Mary satia \\
2 & Bathuri $\times$ BR12 (Mayana) & 49 & Boailla $\times$ Lagi jota \\
3 & Baisamugur X Soda & 50 & Beri $\times$ Mazra \\
4 & Soda $\times$ BR2 (Mala) & 51 & Moush Doll $\times$ Bawoi \\
5 & Beni muri $\times$ BR319-1-HR-12 & 52 & Mazra $\times$ Morich Boti \\
6 & Beni muri $\times$ Bora dhan & 53 & Mazra $\times$ Mi-mandisarang \\
7 & Beni muri $\times$ Agun Ban & 54 & Mazra $\times$ Beri \\
8 & Beni muri $\times$ Bil Kalae & 55 & Mi-Mandi $\times$ Nordi \\
9 & Beni muri $\times$ Borga Dhan & 56 & Mi-Mandi $\times$ BR319-1-HR-12 \\
10 & Beni muri $\times$ Bali Bokri & 57 & Mi-Mandi $\times$ Bar Pa \\
11 & Beni muri $\times$ Soda & 58 & Mary satia $\times$ Bil Kalae \\
12 & BR319-1-HR-12 $\times$ BR1(chandina) & 59 & Noroi $\times$ Bil Kalae \\
13 & Borga Dhan $\times$ Zamir Saita & 60 & Lagi jota $\times$ Boailla \\
14 & Chamka $\times$ Panchash & 61 & Noroi $\times$ Boailla \\
15 & Chamka $\times$ BR1 (chandina) & 62 & Nordi $\times$ Boailla \\
16 & Chamka $\times$ BR2 (Mala) & 63 & Padha Moidu $\times$ BR15 (Mohinye) \\
17 & Boailla $\times$ BR6 & 64 & Panchash $\times$ BR15 (Mohinye) \\
18 & Bhatkarari $\times$ BR7 (Brri Balam) & 65 & Sodai Soru $\times$ BR15 (Mohinye) \\
19 & Atithi dhan $\times$ Soda & 66 & Beni muri $\times$ Padma Moni \\
20 & Gutle $\times$ Soda & 67 & Beni muri $\times$ Rathail \\
21 & Darial $\times$ Sail bogi & 68 & BR319-1-HR-12 $\times$ Pankliiras \\
22 & Holat $\times$ Sail bogi & 69 & Bora dhan $\times$ Padma Moni \\
\hline
\end{tabular}




\begin{tabular}{llll}
\hline Sl \# & Combinations & Sl \# & Combinations \\
23 & Darial $\times$ Tapa sail & 70 & Bora dhan $\times$ Sodai Soru \\
24 & Gutle $\times$ Tapa sail & 71 & Balion $\times$ Padha Moidu \\
25 & Benaful $\times$ Tarabali & 72 & Balion $\times$ Panchash \\
26 & Holat $\times$ Zamir Saita & 73 & Boailla $\times$ Parija \\
27 & Hijoli Aus $\times$ Zamir Saita & 74 & Boailla $\times$ Rathail \\
28 & Hati Bajor $\times$ Tapa sail & 75 & Khushni $\times$ Chitri \\
29 & Hati Bajor $\times$ Zamir Saita & 76 & Bali Bokri $\times$ Mary satia \\
30 & Jamri saity $\times$ Zamir Saita & 77 & Pankliiras $\times$ Borga Dhan \\
31 & Jagli $\times$ Soda & 78 & Borga Dhan X Rathail \\
32 & Kali Bori $\times$ Soda & 79 & Chakulya $\times$ Parangi \\
33 & Katar $\times$ BR8 (Aasa) & 80 & Darial $\times$ Pankliiras \\
34 & Baisamugur $\times$ Kalo Hizli & 81 & Ratol $\times$ Rathail \\
35 & Baisamugur $\times$ Kheri Jamri & 82 & Ratol $\times$ Darial \\
36 & Lagi jota $\times$ Baisamugur & 83 & Barmulka- $\times$ Japanese \#3 \\
37 & Manik Mondol-2 $\times$ BR9 $($ Sufala $)$, & 84 & Benaful $\times$ Joba \\
38 & Padha Moidu $\times$ Panchash & 85 & Panburi $\times$ Japanese \#3 \\
39 & Padha Moidu $\times$ Tepakain & 86 & Soloi, $\times$ Haji Sail \\
40 & Bil Kalae $\times$ Balam & 87 & Soloi $\times$ Hati Bajor \\
41 & Bil Kalae $\times$ Boailla & 88 & Soloi $\times$ Japanese \#7 \\
42 & Bil Kalae $\times$ BR16 $($ Sahya Balam $)$ & 89 & Benaful $\times$ Moush Doll \\
43 & Jamri saity $\times$ BR15 $($ Mohinye $)$ & 90 & Morich Boti $\times$ Benaful \\
44 & Jamurus $\times$ BR16 $($ Sahya Balam $)$ & 91 & Morich Boti $\times$ Benaful \\
45 & Japanese \#3 $\times$ Baismuguria & 92 & Benaful $\times$ Matia \\
46 & Khamar Mundu $\times$ Baismuguria & 93 & Benaful $\times$ Porangi 7 \\
47 & Bawoi $\times$ Laksmi lofa & 94 & Benaful $\times$ Parangi \\
\hline & & & \\
\hline
\end{tabular}

\section{DISCUSSIONS}

In crop improvement breeding program these genetically diverse genotypes could be chosen as parents for crossing program to create genetic variability and produce transgressive segregants. It was also recognized that two Aus landraces (Panchash and Parija) were sorted out as exactly same genotypes in this analysis (zero dissimilarity) might possess same genetic background. Hence, microsatellite marker based molecular fingerprinting could serve as a potential basis in the identification of genetically distant accessions as well as in duplicate sorting of the morphologically close accessions. 


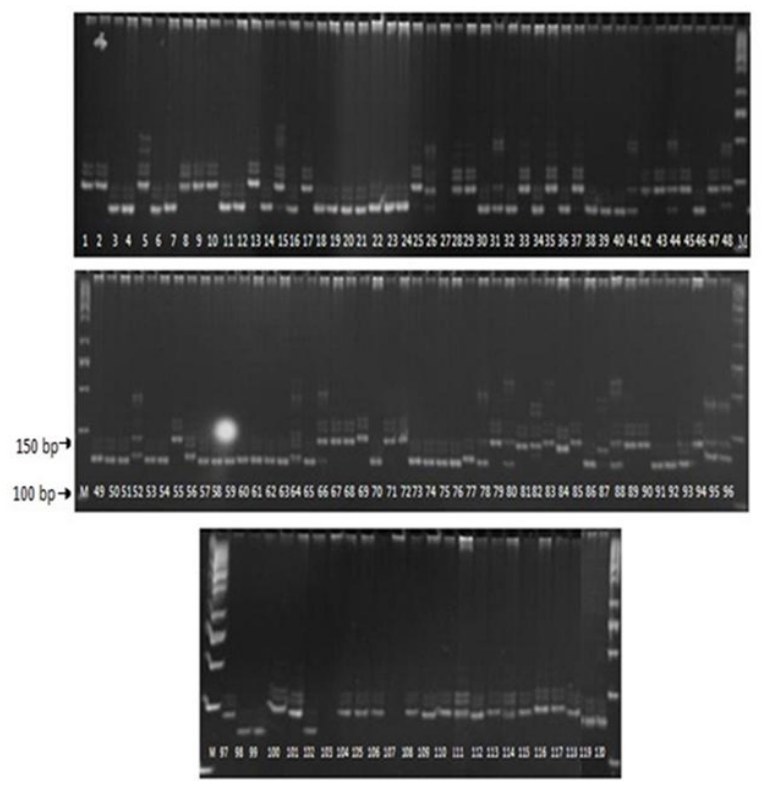

Figure 1: DNA profile of 120 Aus genotypes (108 landraces and 12 BRRI released Aus variety) with the SSR marker RM519

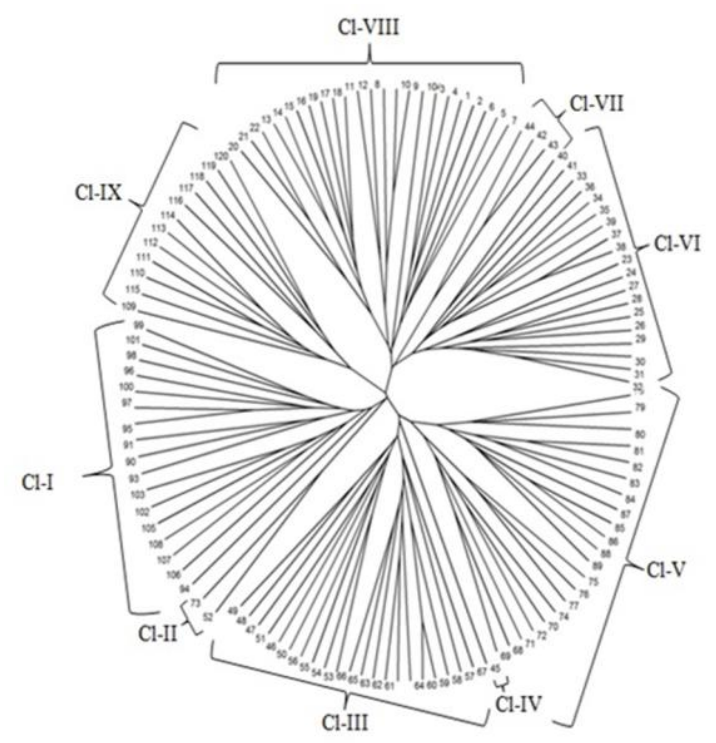

Figure 2: An unrooted neighbor-joining tree showing the genetic relationships among 120 Aus landraces based on the alleles detected by 45 microsatellite markers 
In contrast, DNA-based molecular markers have proven to be powerful tools in the assessment of genetic variation and in the elucidation of genetic relationships within and among species, characterized by abundance and untouched by environmental influence (Powell et al. 1996). Ravi et al. (2003) also generated unique SSR profiles in rice by using a few primers that covered all 12 chromosomes. In the present investigation, SSR marker loci generated by 45 primers were used to assess the genetic diversity among 120 Aus rice genotypes. The SSR primers generated 228 alleles with the number of alleles per locus varying from 6 to 30. Similar number of microsatellite markers was previously used as subset for genetic diversity analysis of O. sativa (Garris et al.; 2005 Chakrabarthia and Naravaneni, 2006). The average number of alleles per locus was 13.0, indicating a greater magnitude of diversity among the plant materials included in this investigation. This value is comparable to 4 alleles (RM484) to 31 alleles (RM474), with an average of 13.0 alleles across the 30 loci (Thomson et al., 2007). The polymorphic information content (PIC) values were ranged 0.5211 (RM536) to 0.9369 (RM519) with an average 0.8217. The PIC values observed, are comparable to three previous estimates of microsatellite analysis in rice viz., o.67-0.88 (Gohain et al., 2006), 0.20-.90 with an average 0.560 (Jain et al., 2003). Many studies have also reported significant differences in allelic diversity among various microsatellite loci (Ravi et al., 2003). The alleles revealed by markers showed a high degree of polymorphism. The mean PIC value observed in this study was higher than the PIC value of 0.578 recorded by Ravi et al. (2003) in an earlier study among rice cultivars, landraces and wild relatives. The findings indicated that the genotypes used in the present study were more diversed due to differences in origin, ecotype and speciation. Panaud et al. (1996) studied using SSR markers in rice, described similarly high genetic similarity among landraces of common geographic origin and low similarity among landraces of diverse geographic origins.

The efficient use of SSR markers to discriminate between Oryza species with various genomes was also demonstrated by Cai and Morishima (2002). The multi allelic nature of SSR markers has the unambiguous advantage of discriminating between the genotypes more precisely. The Unrooted neighbor-joining tree cluster analysis of the SSR-based genetic similarity matrix resulted in the classification of Aus genotypes into separate clusters. Moreover, varietal profiling based on SSR markers will be more reliable as compared to profiling based on other markers, since SSR markers detect finer levels of variations among closely related lines. Cluster I was obtained as largest constellation and included 23 genotypes.

\section{CONCLUSION}

The allelic diversity revealed by 45 SSR primers was sufficient enough to distinguish among the tested Aus rice genotypes. The allelic variation was lower within the genotypes group than the other genotypes, indicating the possibility to exploit distant relatives to broaden the genetic base of rice. 


\section{REFERENCES}

Amanda, J.G., Tai T.H., Coburn, J., Kresovich, S. and McCouch, S. 2004. Genetic Structure and Diversity in Oryza sativa L. Genetics, 169: 1631-1638.

Amanda, I.J. and Rawat, D.S. 1984. Genetic diversity, combining ability and heterosis in brown mustard. Indian Journal of Genetics and Plant Breeding, 44: 226-234.

Cassman, K.G. 1999. Ecological intensification of cereal production systems: Yield potential, Soil quality, and precision agriculture. Proceedings of the National Academy of Sciences (USA), 96: 5952-5959.

Cai, H. and Morishima, H. 2002. Diversity of Rice Varieties and Cropping Systems in Bangladesh Deep water Areas. JARO, 34(4): 225-231.

Chakravarthi, B.K. and Naravaneni, R. 2006. SSR marker based DNA fingerprinting and diversity study in rice (Oryza sativa. L). African Journal of Biotechnology, 5 (9): 684 688

De, R.N., Setharam, R., Sinha, M.K. and Banarjee, S.P. 1988. Genetic divergence in rice. Indian Journal of Genetics and Plant Breeding, 48: 189-194

Dutta, R. K., Lahiri, B. P., Baset and Mian, M. A. 1998. Characterization of some aromatic and fine rice cultivars in relation to their phsico-chemical quality of grains. Indian Journal of plant physiology., 3(1): 61-64

Garris, A.J., Tai, T.H. Coburn, J., Kresovich, S. and Mcouch, H. 2005. Genetic Structure and Diversity in Oryza sativa L. Genetics, 169: 1631-1638

Gohain, B., Talukdar, A. and Modi, M.K. 2006. Mining of allele(s) for aroma in local aromatic rice (joha) germplasm of Assam using SSR marker. Abstracts. 26 ${ }^{\text {th }}$ International Rice Conference-2, International Rice Congress 2006 October 9-13, 2006, New Delhi, India. P: 74

IRGSP, 2005. The map-based sequence of the rice genome. Nature, 436: 793-800

Khush, G.S. 2005. What it will take to feed 5.0 billion rice consumers in 2030. Plant Molecular Biology, 59: 1-6

Liu, K. and Muse, S.V. 2005. Power Marker: Integrated analysis environment for genetic marker data. Bioinformatics, 21: 2128-2129

McCouch, S.R., Teytelman, L., Xu, Y., Lobos, K.B., Clare, K., Walton, M., Fu, B., Maghirang, R., Li, Z., Xing, Y., Zhang, Q., Kono, I., Yano, M., Fjellstorm, R., Declerck, G., Schneider, D., Cartinhour, S., Ware, D. and Stein, L. 2002. Development and mapping of 2240 new SSR markers for rice (Oryza sativa L.). DNA Research, 9: 199-207

Panaud, O., Chen, X. and McCouch, S. R. 1996. Development of microsatellites markers and characterization of simple sequence length polymorphism (SSLP) in rice (O. sativa L.). Molecular Genetics and Genomics, 252:597-607

Peng, S., Cassman, K.G., Virmani, S.S., Sheehy, J. and Khush, G.S. 1999. Yield potential trends of tropical rice since release of IR8 and the challenge of increasing rice yield potential. Crop Science, 39: 1552-1559 
Powell, W., Machray, G.C. and Provan, J. 1996. Polymorphism revealed by simple sequence repeats. Trends Pant Science, 1: 215-222

Ravi, M., Geethanjali, S., Sameeyafarheen, F. and Maheswaran, M. 2003. Molecular Marker based Genetic Diversity Analysis in Rice (Oryza sativa L.) using RAPD and SSR markers. Molecular Marker based Genetic Diversity Analysis in Rice (Oryza sativa L.) using RAPD and SSR markers. Euphytica, 133: 243-252

Rohlf, F. 2002. NTSYS-pc: Numerical taxonomy and multivariate analysis system, 2.2 edn. Department of Ecology and Evaluation, State University of NY, Stony Brook.

Temnykh, S., Clerck, W.D., Carthinour, S., Lukashova, A., lipovich, L. and McCouch, S.R. 2001. Computational and experimental analysis microsatellites in rice (Oryza sativa L.): frequency, length variation, transpose associations and genetic marker potential. Genome Research, 11: 1441-1552

Thomson, M.J., Septiningsih, E.M., Suwardjo, F., Santoso, T.J., Silitonga, T.S. and McCouch, S. R. 2007. Genetic diversity analysis of traditional and improved Indonesian rice (Oryza sativa L.) germplasm using microsatellite markers. Theoretical and Applied Genetics, 114(3): 559-568 